\title{
Improved Antitumor Response to Isolated Limb Perfusion With Tumor Necrosis Factor After Upregulation of Endothelial Monocyte-Activating Polypeptide II in Soft Tissue Sarcoma
}

\author{
T. E. Lans, MD, T. L. M. ten Hagen, Dr, R. van Horssen, Ing, \\ P. C. Wu, MD, S. T. van Tiel, Ing, S. K. Libutti, MD, \\ H. R. Alexander, MD, and A. M. M. Eggermont, MD, Prof Dr
}

\begin{abstract}
Background: Experiments with tumor necrosis factor $\alpha$ (TNF) in rodents have shown that a high dose can lead to hemorrhagic necrosis in tumors. Endothelial monocyte-activating polypeptide II (EMAP-II) is a novel tumor-derived cytokine, and its expression increases the TNF-1 receptor on tumor endothelium, enhances the induction of tissue factor on tumor endothelial cells, and has an antiangiogenic effect. It has recently been shown that in vivo sensitivity of tumor vasculature to TNF is determined by tumor production of EMAP-II.

Methods: We measured the level of EMAP-II in a TNF-resistant soft tissue sarcoma. We subsequently stabile-transfected this cell line with a retroviral construct containing the EMAP gene. In an extremity perfusion model in tumor-bearing rats, we measured response rates to TNF therapy.

Results: Functional EMAP-II production was increased after this transfection. Immunostaining of paraffin-embedded tumor tissue sections in rats showed an overexpression of human EMAP-II. Results of the TNF perfusions in rats suggest that this tumor is more sensitive to TNF therapy.

Conclusions: EMAP-II is produced in various levels. One can increase the sensitivity of tumor for TNF therapy in vivo by upregulating the EMAP-II production. This result leaves an opportunity for enhanced TNF response of tumors in future settings.
\end{abstract}

Key Words: Limb perfusion-EMAP-II-Sarcoma-TNF.

Studies of the antitumor activity of systemically administered tumor necrosis factor (TNF) in humans have been hampered by severe dose-limiting toxicity at relatively low doses of the cytokine which are insufficient for an antitumor response. ${ }^{1}$ This situation changed when TNF was applied in the isolated limb perfusion (ILP) setting. The efficacy of the application of TNF in combination with cytostatic agents in ILP for the treatment of patients with in-transit melanoma

\footnotetext{
Received September 26, 2001; accepted May 14, 2002.

From the University Hospital Rotterdam-Daniel den Hoed Cancer Centre, Department of Surgical Oncology (TEL, TLMtH, RvH, STvT, AMME), Rotterdam, The Netherlands; and Surgery Branch (PCW, SKL, HRA), Center for Cancer Research, National Cancer Institute, Bethesda, Maryland.

Address correspondence and reprints requests to: T. E. Lans, MD, Erasmus University Rotterdam, Laboratory for Experimental Surgical Oncology, Room Ee 110a, PO Box 1738, 3000 DR Rotterdam, The Netherlands; Fax: 00-31-104089471; E-mail: lans@heel.fgg.eur.nl.

Published by Lippincott Williams \& Wilkins @ 2002 The Society of Surgical Oncology, Inc.
}

metastases or locally advanced soft tissue sarcomas has now been well established. ${ }^{1-3}$ This treatment can be used as a limb-sparing neoadjuvant therapy when it is followed by local excision of residual tumor. TNF became a registered cancer drug in Europe on the basis of results obtained in multicenter studies in which clinical response rates of $75 \%$ and limb salvage rates of $71 \%$ were observed in 196 patients who had been classified as having unresectable tumors that could have been managed only by amputation. Approval by the European Agency for the Evaluation of Medicinal Products was given for the use of TNF in the setting of an ILP for locally advanced extremity grade II to III soft tissue sarcomas. ${ }^{4}$ However, $25 \%$ of all patients remain in whom we cannot achieve a sufficient antitumor effect for limb preservation. The answer as to why this group of patients is not responding is not known.

There is evidence that the mechanism of action of TNF is not directly on tumor cells, but that it acts indirectly by destruction of tumor-associated endothelium..$^{5}$ This destruc- 
tion may eventually lead to the death of tumor cells when TNF is used in combination with an antitumor drug. The antitumor effects after TNF-containing ILPs can be extremely rapid, indicating that the TNF-mediated collapse of the tumor vascular bed may play an essential role in the antitumor mechanism. The selective destructive effects of TNF ILP on tumor-associated vessels have been illustrated in the clinic by means of pre- and postperfusion angiography. ${ }^{1}$ Severe hemorrhagic necrosis accompanied with vascular destruction was observed after ILP with TNF in combination with melphalan.

Our laboratory has developed ILP models with different tumors in rats to study prerequisites for improving efficacy and to study mechanisms of synergy.6,7 These models mimic the clinical setting closely with respect to response rate and histopathology. The crucial observation has been that TNF selectively and highly significantly enhances the uptake of melphalan and doxorubicin in the tumors. ${ }^{6}$

Endothelial monocyte-activating polypeptide II (EMAPII) is a tumor-derived cytokine with proinflammatory properties. It induces procoagulant activity on the surface of endothelial cells and monocytes/macrophages in vitro, as well as upregulation of E- and P-selectin expression and the ability to upregulate TNF receptor-1 expression on tumor vasculature. Furthermore, EMAP-II induces apoptosis and has antiangiogenic effects. ${ }^{1}$ These data led us to hypothesize that EMAP-II could be of additional benefit in the TNF perfusion circuit. Wu et al. ${ }^{8}$ defined a way to upregulate EMAP-II production by means of retroviral transfection in a melanoma cell line. We used this method of transfection on a TNF-resistant soft tissue sarcoma. We describe here the application of the sensitizing gene therapy and subsequent regional TNF treatment and its effects on in vivo tumor growth. Because EMAP-II can have additional benefits in long-term anticancer treatment regarding its antiangiogenic effect (e.g., to prevent outgrowth of neovasculature and metastases) and is toxic when injected systemically, a long-lasting and local production of EMAP-II in a tumor should be favorable. This can be maintained after retroviral transfection and subsequent upregulation of the protein synthesis by tumor cells. It was our goal to identify factors of TNF resistance or sensitivity and the potential role of EMAP-II in this.

\section{MATERIALS AND METHODS}

\section{Animals}

Inbred male Brown Norway (BN) rats, weighing 200 to $300 \mathrm{~g}$, were obtained from Harlan-CPB (Austerlitz, The Netherlands). All animals were kept at standard laboratory conditions and were fed a standard laboratory diet (Hope
Farms, Woerden, The Netherlands). The experimental protocols adhered to the rules laid down in the Dutch Animal Experimentation Act (1977) and the published Guidelines on the Protection of Experimental Animals by the council of the European Community (1986). All animal studies were performed in accordance with protocols approved by the Animal Care Committee of the Erasmus University of Rotterdam, The Netherlands.

\section{Tumor Cell Lines}

$\mathrm{BN}-175$ is a nonimmunogenic, rapidly growing soft tissue sarcoma syngeneic in $\mathrm{BN}$ rats, with a tumor doubling time of approximately 2 to 3 days. This tumor was implanted into the flank of donor rats and passaged serially. For this study we used small tumor fragments $\left( \pm 1 \mathrm{~mm}^{2}\right)$ that were subcutaneously implanted into the right hind limb just above the ankle. All surgical interventions were performed at a tumor diameter of 5 to $10 \mathrm{~mm}$, at least 7 days after implantation. Rats were killed if tumor diameter exceeded 25 $\mathrm{mm}$. The human umbilical vein endothelial cells (HUVECs) used were obtained from BioWhittaker Europe (Verviers, Belgium) and consisted of pooled cells by multiple donors.

\section{Drugs}

Recombinant human TNF- $\alpha\left(4.9-5.8 \times 10^{7} \mathrm{U} / \mathrm{mg}\right)$, recombinant human EMAP-II (hEMAP-II; $3.85 \mu \mathrm{g} / \mathrm{mL}$ ), and antibodies directed to hEMAP-II were provided as a kind gift by Dr. G. Adolf (Boehringer Ingelheim GmbH, Vienna, Germany).

\section{Transfection of BN-175 Tumor Cells With the EMAP-II Gene}

Viral supernatant from the Gibbon Ape ecotropic packaging cell line PG-WU was used to transfect the BN-175 tumor cell line. Tumors were grown in six-well plates to approximately $40 \%$ confluence. The viral supernatant was then collected and filtered across a $.45-\mu \mathrm{m}$ low-protein-binding membrane (Corning Costar, Cambridge, MA), and polybrene (Sequa Brene; Sigma, St. Louis, MO) was added at a concentration of $1 \mu \mathrm{l} / \mathrm{mL}$ of supernatant. Tumor cells were washed twice with phosphate-buffered saline (PBS) and incubated for 6 hours on 3 subsequent days in an incubator at $37^{\circ} \mathrm{C}$ and $5 \% \mathrm{CO}_{2}$. In between incubation with viral supernatant, cells were given normal medium to recover. After 5 to 7 days, the transfected tumor cells (named BN-E) were placed into $75-\mathrm{cm}^{2}$ flasks and expanded under neomycin selection at $800 \mu \mathrm{g} / \mathrm{mL}$. 


\section{Detection of Incorporation of hEMAP in Genomic DNA of Rat Tumor Cells by PCR}

Transfected tumor cells growing in $800 \mu \mathrm{g} / \mathrm{mL}$ of neomycin were trypsinated and pelleted. DNA was extracted with the QIamp DNA minikit (Qiagen Inc., Valencia, CA) and amplified by PCR by using primers specific for both a part of the EMAP insert and the downstream retroviral internal ribosome entry site region. For EMAP, the following primers were used: 5'AATCGGATGGTGATTTTACTTTGTA-3' at both times as the forward primer and backward; 5'-CATTTTATTTGATTCCACTGTTGC-3' with a PCR product of 333 base pairs (bp) for the EMAP part; and backward for the internal ribosome entry site and EMAP 5'-GAATGCTCGTCAAGAAGACAG-3', providing a 577-bp PCR product. PCR amplification was performed under the following conditions: 5 minutes at $95^{\circ} \mathrm{C}, 35$ cycles $\left(94^{\circ} \mathrm{C}\right.$ for 45 seconds, $55^{\circ} \mathrm{C}$ for 45 seconds, and $72^{\circ} \mathrm{C}$ for 60 seconds); and 7 minutes at $72^{\circ} \mathrm{C}$, soak at $4^{\circ} \mathrm{C}$. Amplified PCR products were subjected to gel electrophoresis on a $1 \%$ agarose gel containing ethidium bromide.

\section{Western Blot Analysis of Transfected Tumor Cells for EMAP}

After transfection of the tumor cells, cells were collected, washed, and centrifuged. Cell pellets were lysed at $4^{\circ} \mathrm{C}$ in a buffer containing $50 \mathrm{mM}$ TrisCl (pH 8.0), 150 $\mathrm{mM} \mathrm{NaCl}, .02 \%$ Na-azide, $.1 \%$ sodium dodecyl sulfate, $1 \mu \mathrm{g} / \mathrm{mL}$ of aprotinin, $5 \%$ Na-deoxycholate, $1 \%$ Nonidet P-40 (Sigma-Aldrich, Zwijndrecht, The Netherlands), deoxyribonuclease I $10 \mathrm{ng} / \mathrm{mL}$, and $100 \mu \mathrm{g} / \mathrm{mL}$ of phenylmethyl sulfonyl fluoride for 10 minutes. Samples were vortexed, and debris was pelleted by centrifugation at $15,000 \times g$ for 10 minutes. Supernatant protein content was determined by BCA protein assay (Pierce Chemical Co., Rockford, IL), and a final loading concentration of $120 \mu \mathrm{g}$ per sample was used. Lysates were mixed with Laemmli buffer containing $5 \% \beta$-mercaptoethanol, boiled for 5 minutes, and electrophoresed on a $15 \%$ acrylamide gel by using sodium dodecyl sulfate polyacrylamide gel electrophoresis. The membranes were subsequently blocked for nonspecific binding overnight at $4{ }^{\circ} \mathrm{C}$ with $\mathrm{PBS} / 5 \%$ nonfat dry milk/.05\% Tween and incubated for 2 hours with EMAP-II polyclonal rabbit antiserum (diluted 1/2000 in PBS/5\% nonfat dry milk $/ .05 \%$ Tween). After washing thoroughly, the membranes were incubated for 1 hour with biotinylated goat anti-rabbit immunoglobulin $\mathrm{G}$ antiserum (diluted 1/3000 in PBS/5\% nonfat dry milk/.05\% Tween). Membranes were again washed and incubated with alkaline phosphatase-labeled streptavidin and developed with en- hanced chemiluminescence by adding bromo-cloroindolyl-phosphate/nitro-blue-tetrazolium.

\section{Coagulation Assay}

Conditioned medium from wild-type BN-175 and BN-E tumor cell lines was tested for procoagulant activity by using a two-stage coagulation assay. HUVECs were grown to $\sim 80 \%$ confluence in six-well multiwell tissue culture plates in complete endothelial growth media (Cambrex Bio Science, Baltimore, MD). Cells were washed twice with sterile PBS. Tumor-conditioned medium was produced by growing $\sim 5 \times 10^{6}$ tumor cells per $75-\mathrm{cm}^{2}$ tissue culture flask in $15 \mathrm{~mL}$ of serum-free medium. After 24 hours, conditioned medium was collected and vacuum-filtered across a $.45-\mu \mathrm{m}$ membrane to remove cellular debris and was used directly. HUVECs were treated at a concentration of $1 \mathrm{~mL}$ of conditioned medium per well. Normal medium was used as a negative control, and TNF- $\alpha$ at a concentration of $1 \mu \mathrm{g} / \mathrm{mL}$ of medium was used as a positive control. The samples were incubated for 6 hours at $37^{\circ} \mathrm{C}$ in a $5 \% \mathrm{CO}_{2}$ incubator and assayed for procoagulant activity. Medium was removed from the treated HUVECs, and cells were washed with $\mathrm{Ca} / \mathrm{Mg}$-free PBS. After 10 minutes of incubation with $1 \mathrm{~mL}$ of $25 \mathrm{mM}$ Tris/ethylenediaminetetraacetic acid ( $\mathrm{pH}$ 7.4) per well, cells could be gently removed from the wells and subsequently collected and centrifuged at $14,000 \times g$ for 10 minutes at room temperature. Supernatant was carefully removed, and the resultant cell pellet was resuspended in a $100-\mu l$ assay buffer (buffer: $250 \mu \mathrm{l}$ of $1 \mathrm{M}$ Tris; $300 \mu \mathrm{l}$ of $5 \mathrm{M} \mathrm{NaCl}$; $10 \mathrm{mg}$ of $.1 \%$ bovine serum albumin; distilled $\mathrm{H}_{2} \mathrm{O}$ to 10 $\mathrm{mL}$ ) and shortly vortexed. Cell pellet was added to 100 $\mu \mathrm{l}$ of human citrated plasma (Biopool, Venture, CA), mixed well, and incubated at $37^{\circ} \mathrm{C}$ for 3 minutes in a clinical fibrometer. The coagulation reaction was catalyzed by adding $100 \mu \mathrm{l}$ of $30 \mathrm{mM} \mathrm{CaCl}_{2}$ (Sigma), and clotting time was measured. Standard curves were generated by using lipidated recombinant human tissue factor (American Diagnostica, Greenwich, CT).

\section{Immunohistochemical Staining of hEMAP in Rat Tumor Tissue}

Tumor was induced by subcutaneous injection of $\sim 2 \times$ $10^{6} \mathrm{BN}$ or BN-E tumor cells in the flank region of syngeneic BN rats. When the tumor reached $10 \mathrm{~mm}$ in diameter, it was resected from the animal, immediately fixated in $4 \%$ formalin, and embedded in paraffin. Assessment of tumor morphology was performed on 5- to $8-\mu \mathrm{m}$ hematoxylin and eosin-stained sections. Before staining for EMAP-II, sections were dewaxed with xylene and rehydrated by passing through a graded series of 
alcohol (100\%-70\%) followed by PBS. Sections were microwaved two times for 5 minutes in $.01 \mathrm{M}$ citrate buffer $(2.10 \mathrm{~g} / \mathrm{L}$ of citric acid and $1 \mathrm{~g} / \mathrm{L}$ of sodium hydroxide) to enhance immunoreactivity. All incubations were performed in a humidified chamber at room temperature. Sections were incubated in 5\% normal goat serum for 30 minutes to block nonspecific binding of antibodies. Sections were rinsed with PBS/Tween, and rabbit polyclonal antibody against hEMAP-II was added at a 1/100 dilution. Polyclonal rabbit immunoglobulin G (Santa Cruz Biotechnology, Santa Cruz, CA) was used as a negative control. Sections were incubated for 1 hour, washed three times with PBS, and subsequently incubated with goat anti-rabbit secondary antibody conjugated to peroxidase for 1 hour. After washing three times in PBS, slides were incubated with the avidin-biotin complex reagent, followed by diaminobenzidine substrate. Thereafter the slides were rinsed with distilled water and counterstained with hematoxylin. Finally, slides were dehydrated with graded alcohol and xylene and covered with DPX. Sections were examined with a Leica (Wetzlar, Germany) DM-RXA microscope, and images were made by using a Sony (Tokyo, Japan) DXC950 digital camera.

\section{ILP Model}

The perfusion technique was performed as described previously. $6,7,9$ In our laboratory, Manusama et al. ${ }^{7}$ modified the technique of ILP in rats, originally described by Benckhuijsen et al. ${ }^{10}$ In this model, by using the BN-175 soft tissue sarcoma, it was shown that only when TNF was combined with melphalan or doxorubicin could a tumor response be reached. In comparison to the clinical setting, our rat model demonstrated partial or complete regression of the tumor in approximately $75 \%$ of rats receiving an ILP with melphalan plus TNF. We used the BN-175 soft tissue sarcoma to set up an experimental environment where we could improve the response rate to TNF.

Briefly, small fragments $(3-5 \mathrm{~mm})$ of tumor were implanted subcutaneously into the right hind limb of the rat. Approximately 7 to 8 days after implantation, tumors reached an average diameter of 8 to $12 \mathrm{~mm}$, and then treatment was started. Animals were anesthetized with Hypnorm (Janssen Pharmaceutica, Tilburg, The Netherlands) and ketamine (Apharmo BV, Arnhem, The Netherlands). Heparin (50 IU) was injected intravenously to prevent coagulation. To keep the rat's hind limb at a constant temperature, a warm-water mattress was applied. Temperature was measured with a temperature probe on the skin covering the tumor and was maintained between $38^{\circ} \mathrm{C}$ and $39^{\circ} \mathrm{C}$. The femoral artery and vein were cannulated with silastic tubing (.012-inch inner diameter, .025-inch outer diameter; and .025-inch inner diameter, .047 inch outer diameter, respectively; Dow Corning, Midland, MI). Collaterals were occluded by a groin tourniquet, and the time of isolation started when the tourniquet was tightened. An oxygenation reservoir and a roller pump were included into the circuit. The perfusion solution consisted of $5 \mathrm{~mL}$ of Hemaccel (Behring Pharma, Amsterdam, The Netherlands) in the sham perfusions, and TNF (50 $\mu \mathrm{g})$ was added to the oxygenation reservoir as a bolus in the treatment group. A roller pump (type $505 \mathrm{U}$; Watson Marlow, Falmouth, UK) recirculated the perfusate at a flow rate of $2.4 \mathrm{~mL} / \mathrm{min}$. A washout with $5 \mathrm{~mL}$ of oxygenated Hemaccel was performed at the end of the perfusion. After the perfusion, the cannulas were removed, and the femoral vessels of the perfused limb were ligated. The extensive collateral circulation was capable of restoring the blood supply of the perfused leg.

The perfusion with the wild-type tumor took place 16 hours after intravenous injection with recombinant EMAP-II. In these experiments, $50 \mu \mathrm{g}$ of recombinant EMAP-II, dissolved in $200 \mu \mathrm{l}$ of PBS, was injected intravenously. Rats were closely monitored afterward, and moderate but reversible toxicity was observed after IV administration. Subsequent tumor growth was recorded daily by caliper measurement. Tumor volume was calculated as $.4 \times\left(A^{2} \times B\right)$, where $B$ represents the longest diameter and $A$ the diameter perpendicular to $B$.

\section{RESULTS}

\section{Transfection of the EMAP-II Gene}

Rat soft tissue sarcoma cells were transfected in vitro with retroviral particles containing the $\mathrm{P}$. Wu construct (BN-E) and seemed resistant to continuous exposure with $800 \mu \mathrm{g} / \mathrm{mL}$ of neomycin, demonstrating effective transfection with the construct (data not shown). After 2 weeks of growing in selection media, genomic DNA was extracted from the transfected tumor cell lines. PCR analysis of expression of the EMAP-II gene proved positive for the BN-E cell line (data not shown). Western blot analysis of $120 \mu \mathrm{g}$ of protein of cell lysate of wild-type and transfected tumor cells, compared with $100 \mathrm{ng}$ of recombinant EMAP-II, demonstrated a band corresponding to the size of the human precursor EMAPII, $\sim 34 \mathrm{kDa}$ (Fig. 1). We show only the results with normally growing tumor cells. In our ongoing experiments, we would like to demonstrate the generation of active EMAP-II ( $\sim 22 \mathrm{kDa})$ that was used as a control in these experiments, and we would like to show intermediate cleavage products by inducing apoptosis in the 


\section{PS C BN BN-E}

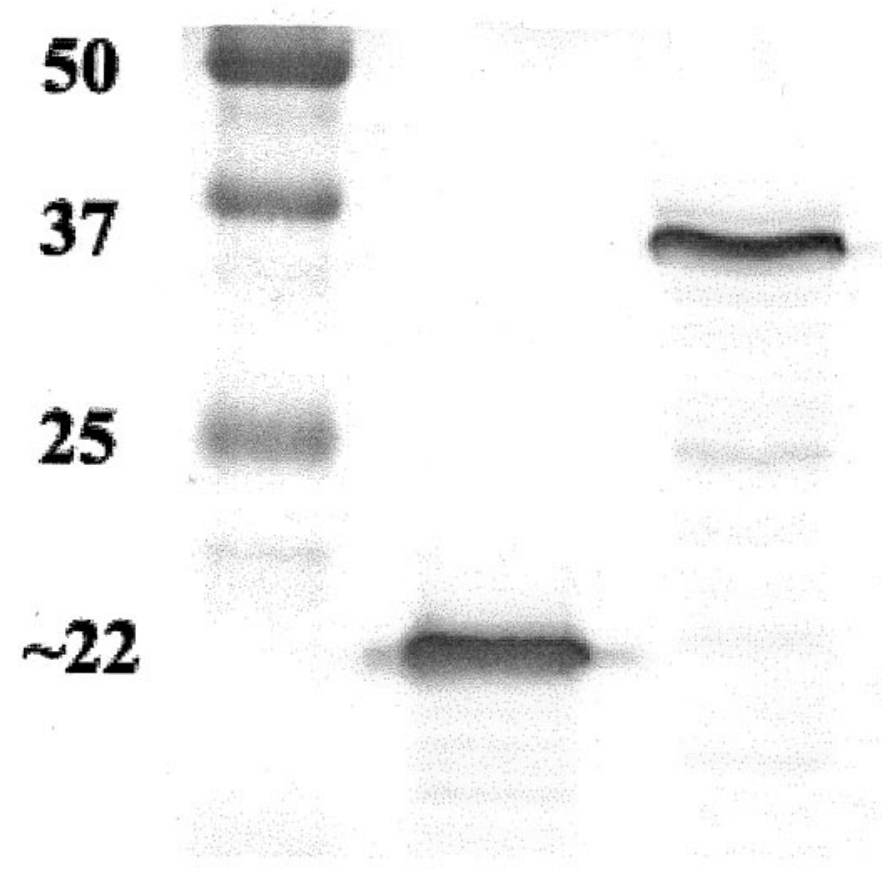

tumor cells. However, this expression seems to be very unstable and transient and shows tremendous variation.

\section{Coagulation Assay}

We wanted to test whether the EMAP-II-transfected cell line, BN-E, produced functional protein. We used a functional bioassay of EMAP-II activity based on the induction of tissue factor expression on human endothelial cells. HUVECs were treated with conditioned medium of transfected tumor cells. As shown in Fig. 2, a 6-hour exposure of HUVECs to medium conditioned by tumor transfected with EMAP-II (BN-E) induced a $20 \%$ increase in the production of tissue factor by these cells, as compared with treatment with conditioned medium from wild-type BN or medium alone.

\section{Immunohistochemical Staining of hEMAP in Rat Tumor Tissue}

The tumor model used for our in vivo studies was generated by subcutaneous injection of $2 \times 10^{6}$ transfected tumor cells in the hind limb of a syngeneic BN rat. Tumors were grown to an average diameter of 8-12 $\mathrm{mm}$, and we observed a growth rate corresponding to a wild-type tumor. As shown in Fig. 3, cells stained positive for EMAP-II as compared with controls. On the hematoxylin and eosin staining (Fig. 4), the morphology of the transfected tumor

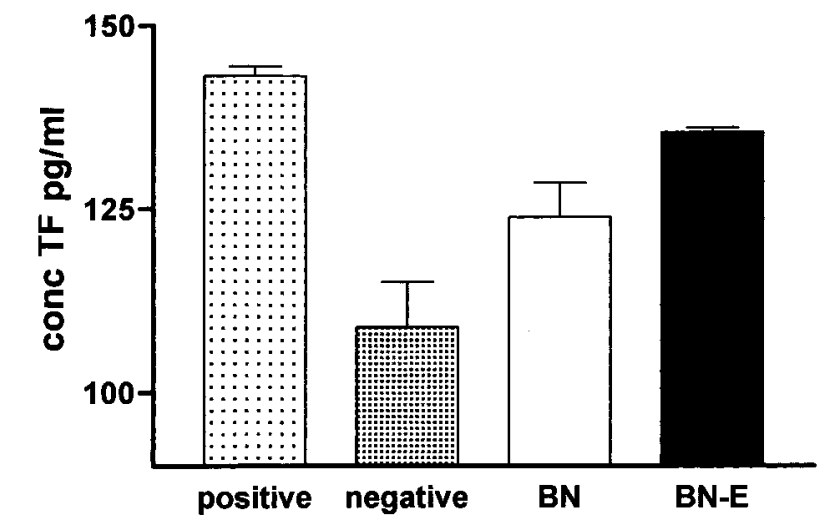

FIG. 2. Tissue factor assay. Lane 1: Positive control; medium of cells treated with $1 \mu \mathrm{g}$ of tumor necrosis factor $\alpha$. Lane 2: Medium of untreated cells. Lane 3: Wild-type tumor medium (BN). Lane 4: Transfected tumor medium (BN-E). Conc TF, concentration of recombinant human tissue factor (American Diagnostica, Greenwich, CT).

was altered with a tendency to more apoptotic cells. This, however, was not reflected in the growth rate of the BN-E. We regard this as apoptosis being induced by the increased EMAP-II production of this tumor.

\section{ILP in Rats With Transfected Tumor}

In Figure 5, the results of TNF ILP in rats bearing the $\mathrm{BN}-\mathrm{E}$ tumor compared with the wild-type tumor are 

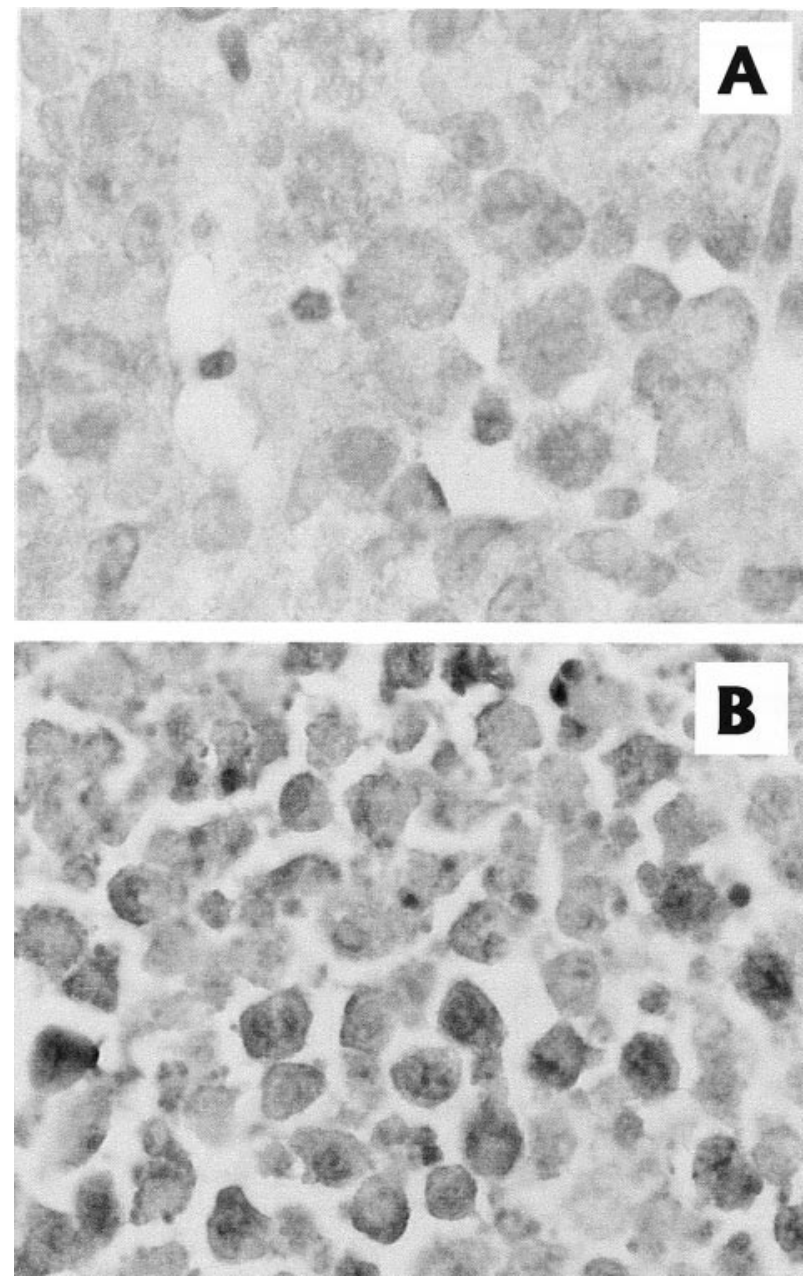

FIG. 3. Immunohistochemical staining with human endothelial monocyte-activating polypeptide II (hEMAP-II) $(\times 100)$. (A) Nontransfected tumor. (B) Marked upregulation of the intracellular present hEMAP-II.

shown. Perfusion with Hemaccel alone resulted in progressive disease in all animals. Although ILP with TNF resulted in a slight inhibition of tumor growth of the wild-type BN tumor compared with the sham control, there was no significant difference in tumor response. As can be seen in this graph, the EMAP-II-transfected tumor BN-E responded significantly better to ILP with 50 $\mu \mathrm{g}$ of TNF. We saw macroscopically a more profound necrosis in the BN-E tumor that led to a significantly slower outgrowth of tumor after the perfusion. Even in animals with transfected tumors, a small rim of viable tumor cells survived. However, as can be seen at day 9 , the overall observed response rate still implied a decline in tumor dimension, whereas profound recurrent tumor growth was demonstrated in all animals from day 11 .

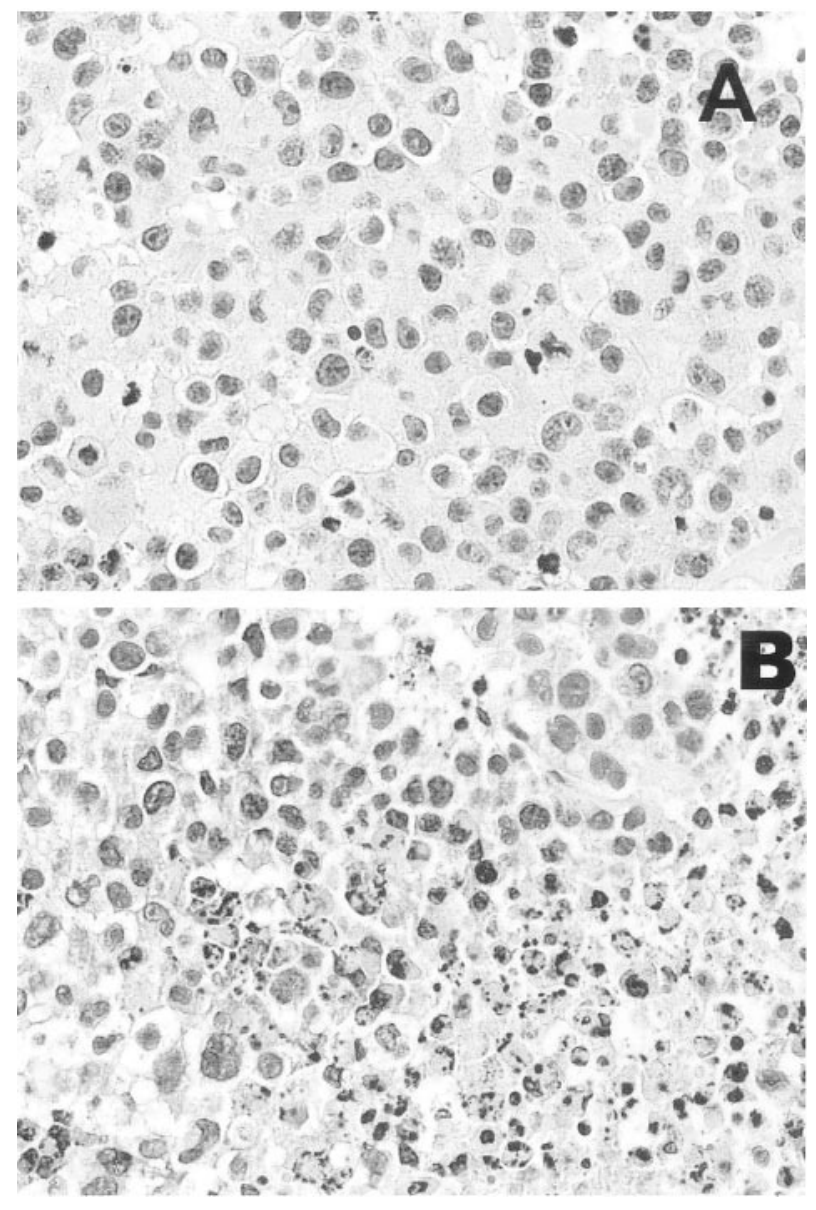

FIG. 4. Hematoxylin and eosin-stained tumor sections $(\times 100)$. $($ A $)$ Normal morphology of wild-type viable tumor cells. (B) Transfected tumor is shown with an aspect of more profound necrosis, disruption of cell structure, and smaller cells.

\section{ILP in Rats Preceded by Recombinant EMAP-II Injection}

A total of $50 \mu \mathrm{g}$ of recombinant EMAP-II was injected intravenously into rats 16 hours before TNF perfusion. Rats showed a moderate toxic response immediately after injection; nevertheless, this was reversible within 4 hours. Figure 6 shows the tumor volume of animals treated with TNF perfusion after pretreatment with recombinant EMAP-II. Sham perfusions after EMAP-II injection were also performed. From the second day, a significant difference in tumor volume was observed in TNF-treated animals bearing the BN-E versus wild-type tumor. Tumors treated with sham perfusion or TNF ILP alone continued to grow; the recombinant EMAP-II pretreated animals showed a delay in tumor growth after TNF ILP. This difference was observed until the ninth day, when both groups started to develop the same growth rate. Both wild-type and BN-E tumors 


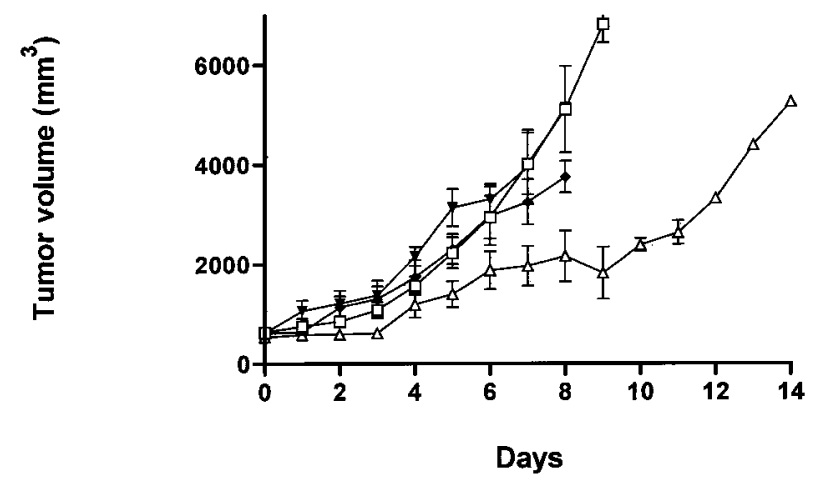

FIG. 5. Growth curves of wild-type tumor after isolated limb perfusion (ILP) with sham $(\square)(\mathrm{n}=5)$ and tumor necrosis factor (TNF) (ム) $(\mathrm{n}=7)$ and of transfected tumor after ILP with sham $(\boldsymbol{\nabla})(\mathrm{n}=7)$ and $\operatorname{TNF}(\triangle)(\mathrm{n}=5)$

showed the same growth rate in the presence of systemically administered recombinant EMAP-II alone.

\section{DISCUSSION}

The results presented in this study show that transfection with EMAP-II of the TNF-resistant tumor BN-175 will render this tumor TNF sensitive and responsive to ILP treatment with TNF. This approach could enhance efficacy in the clinical setting, where $25 \%$ of patients with locally advanced limb-threatening extremity soft tissue sarcoma do not respond to TNF plus chemotherapy in the ILP setting for limb salvage to be achieved.

By introducing isolated perfusion as a mode to apply TNF in patients, we have been able to achieve effective concentrations of TNF in the tumor region. Antitumor effects after TNF-containing ILPs can be extremely rapid. This indicates that also in humans the TNF-mediated collapse of the tumor vascular bed plays an essential role in the antitumor mechanism, as has been illustrated in publications on clinical studies by pre- and postperfusion angiography. ${ }^{11}$ Moreover, in studies in sarcoma patients with magnetic resonance spectrometry, we have clearly shown that the metabolic shutdown of the tumor is virtually complete within 16 hours after the perfusion, confirming the likelihood of TNF mediating its most important effects on the vasculature of the tumor. ${ }^{11}$ At the histopathologic level, these intravascular effects - such as thrombocyte aggregation, erythrostasis, and endothelial and vascular destruction-have been described for the early-stage changes after ILP, which may closely resemble observations made in experimental tumor systems. ${ }^{11}$

Because TNF is known to act on the tumor endothelium, a range of cytotoxic agents have been used in combination to have an additional antitumor effect. After

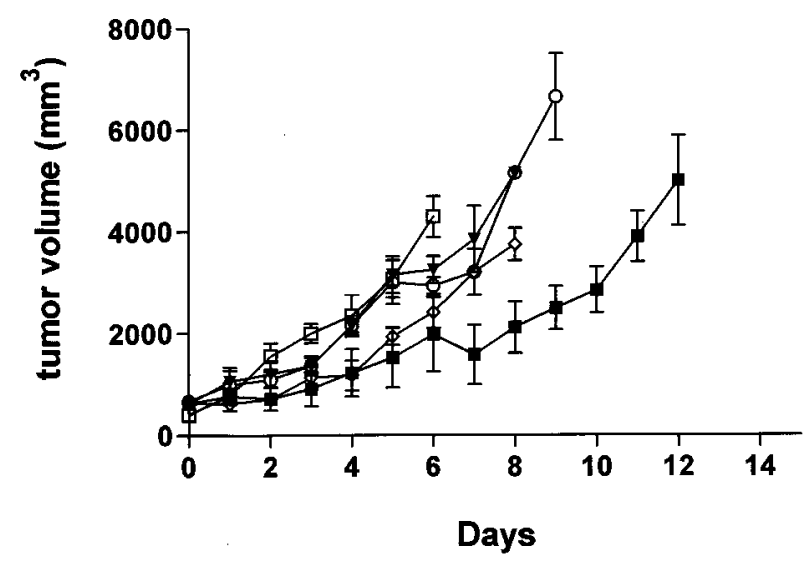

FIG. 6. Results of isolated limb perfusion (ILP) in wild-type (BN) tumor. Control tumor $(\square), \mathrm{n}=6$; sham perfusion, $(\diamond) \mathrm{n}=5$; tumor necrosis factor $(\mathrm{TNF})(\mathbf{\Lambda}), \mathrm{n}=7$; endothelial monocyte-activating polypeptide II (EMAP-II) followed by sham perfusion (O); and ILP with EMAP-II plus TNF $(\square), \mathrm{n}=6$.

the initial destruction of tumor vessels, these agents can exert their toxic effect on the tumor cells.

We introduce here an approach based on a different mechanism to improve the effect of TNF therapy. After the observation that varying tumors respond in a distinct manner to TNF, a cytokine that contributes to this response was isolated. This cytokine, EMAP-II, is produced by tumors and influences the formation of neovasculature in a number of ways, one of them being the upregulation of TNF receptor-1 on tumor-associated endothelial cells. This is an important finding regarding the use of TNF as anticancer therapy.

Therefore, we investigated whether we could enhance the TNF sensitivity of a nonresponding rat sarcoma in vivo by transfecting the tumor with EMAP-II, to increase its endogenous EMAP-II content. EMAP-II is a tumorderived cytokine with proinflammatory properties, inducing procoagulant activity on the surface of endothelial cells and monocytes/macrophages in vitro, as well as upregulation of E- and P-selectin expression and the ability to upregulate TNF receptor-1 expression on tumor vasculature. ${ }^{12}$ The distribution of EMAP-II protein is relatively restricted and is usually associated with tissues that display high turnover and high levels of protein synthesis, e.g., tumor tissue. ${ }^{13}$ It was first isolated from the conditioned medium of the murine methylcholantrene A-induced fibrosarcoma. This tumor responds well to TNF therapy, and investigators found that in the supernatant of this tumor, the cytokine EMAP-II was excreted. Studies have shown that EMAP-II is capable of sensitizing resistant tumors to the antitumor effects of TNF. ${ }^{14,15}$ In response to EMAP-II exposure, endothelial cells are activated, and the expression of TNF receptor-1 
is upregulated, an effect that is known to take place between 16 and 24 hours after exposure to EMAP-II. ${ }^{15}$ It led us to hypothesize that EMAP-II could be of additional benefit in the TNF perfusion circuit. This enabled us to create a more sensitive tumor to TNF therapy, without the toxic side effects of systemic EMAP-II application. In our next experiments, we injected recombinant hEMAP into rats that were 16 hours later subjected to an ILP system with TNF. With a dose of $50 \mu \mathrm{g}$ of recombinant EMAP per rat combined with $50 \mu \mathrm{g}$ of TNF, we saw a significant decrease of growth of the tumors, with an immediate, although moderate and reversible, toxic reaction to the injected EMAP-II. These results were comparable with the effect seen in the transfected tumor and suggest that this effect is mediated by EMAP-II, rather than being an effect created by transfection of the tumor or an effect seen because of varying levels of EMAP-II receptors.

ILP is an interesting method for new treatment modalities, such as adenoviral vector-mediated gene therapy. ${ }^{16,17}$ In our laboratory, we have shown that ILP is the best and most selective method for effective homogeneous transvascular local gene delivery by using adenoviral vectors. Experiments in our soft tissue sarcoma and osteosarcoma ILP models have clearly shown this by making use of luciferase-marker gene and $L a c Z$ gene methodology. ${ }^{18}$ Moreover, we have shown that ILP with adenoviral vector gene delivery of the cytokine interleukin-3 is the only method in these tumor models to achieve good tumor responses, in contrast to the failure of other methods, such as intravenous administration, intra-arterial administration, or intratumoral administration. ${ }^{17}$ It demonstrates that ILP is a valuable method to treat advanced limb tumors and to develop new treatment modalities.

The possibility of manipulating the sensitivity for TNF in a tumor in vivo also leaves a window for systemic application of TNF in future settings. If noninvasive vectors can increase tumor sensitivity in a way that the TNF dose can be decreased, we might be able to save patients in the future by the invasive perfusion procedure and be able to administer TNF systemically.

\section{REFERENCES}

1. Asher A, Mule JJ, Reichert CM, Shiloni E, Rosenberg SA. Studies on the anti-tumor efficacy of systemically administered recombinant tumor necrosis factor against several murine tumors in vivo. J Immunol 1987;138:963-74.

2. Lienard D, Eggermont AM, Koops HS, et al. Isolated limb perfusion with tumor necrosis factor-alpha and melphalan with or with- out interferon-gamma for the treatment of in-transit melanoma metastases: a multicentre randomized phase II study. Melanoma Res 1999;9:491-502.

3. Vaglini M, Belli F, Ammatuna M, et al. Treatment of primary or relapsing limb cancer by isolation perfusion with high-dose alphatumor necrosis factor, gamma-interferon, and melphalan. Cancer 1994;73:483-92.

4. Eggermont AM, Schraffordt KH, Lienard D, et al. Isolated limb perfusion with high-dose tumor necrosis factor-alpha in combination with interferon-gamma and melphalan for nonresectable extremity soft tissue sarcomas: a multicenter trial. J Clin Oncol 1996;14:2653-65.

5. Berger AC, Alexander HR, Wu PC, et al. Tumor necrosis factor receptor I (p55) is upregulated on endothelial cells by exposure to the tumor-derived cytokine endothelial monocyte-activating polypeptide II (EMAP-II). Cytokine 2000;12:992-1000.

6. de Wilt JH, Manusama ER, van Tiel ST, van Ijken MG, ten Hagen TL, Eggermont AM. Prerequisites for effective isolated limb perfusion using tumor necrosis factor alpha and melphalan in rats. Br J Cancer 1999;80:161-6.

7. Manusama ER, Stavast J, Durante NM, Marquet RL, Eggermont AM. Isolated limb perfusion with TNF alpha and melphalan in a rat osteosarcoma model: a new anti-tumor approach. Eur J Surg Oncol 1996;22:152-7.

8. Wu PC, Alexander HR, Huang J, et al. In vivo sensitivity of human melanoma to tumor necrosis factor (TNF)-alpha is determined by tumor production of the novel cytokine endothelial-monocyte activating polypeptide II (EMAPII). Cancer Res 1999;59:205-12.

9. Nooijen PT, Manusama ER, Eggermont AM, et al. Synergistic effects of TNF-alpha and melphalan in an isolated limb perfusion model of rat sarcoma: a histopathological, immunohistochemical and electron microscopical study. Br J Cancer 1996;74:1908-15.

10. Benckhuijsen C, Varossieau FJ, Hart AA, Wieberdink J, Noordhoek J. Pharmacokinetics of melphalan in isolated perfusion of the limbs. J Pharmacol Exp Ther 1986;237:583-8.

11. Olieman AF, Lienard D, Eggermont AM, et al. Hyperthermic isolated limb perfusion with tumor necrosis factor alpha, interferon gamma, and melphalan for locally advanced nonmelanoma skin tumors of the extremities: a multicenter study. Arch Surg 1999; 134:303-7.

12. Berger AC, Alexander HR, Tang G, et al. Endothelial monocyte activating polypeptide II induces endothelial cell apoptosis and may inhibit tumor angiogenesis. Microvasc Res 2000;60:70-80.

13. Murray JC, Barnett G, Tas M, et al. Immunohistochemical analysis of endothelial-monocyte-activating polypeptide-II expression in vivo. Am J Pathol 2000;157:2045-53.

14. Kao J, Ryan J, Brett G, et al. Endothelial monocyte-activating polypeptide II. A novel tumor-derived polypeptide that activates host-response mechanisms. J Biol Chem 1992;267:20239-47.

15. Marvin MR, Libutti SK, Kayton M, et al. A novel tumor-derived mediator that sensitizes cytokine-resistant tumors to tumor necrosis factor. J Surg Res 1996;63:248-55.

16. de Roos WK, de Wilt JH, van Der K, et al. Isolated limb perfusion for local gene delivery: efficient and targeted adenovirus-mediated gene transfer into soft tissue sarcomas. Ann Surg 2000;232:814-21.

17. de Wilt JH, Bout A, Eggermont AM, et al. Adenovirus-mediated interleukin 3 beta gene transfer by isolated limb perfusion inhibits growth of limb sarcoma in rats. Hum Gene Ther 2001;12:489-502.

18. Manusama ER, de Wilt JH, ten Hagen TL, Marquet RL, Eggermont AM. Toxicity and antitumor activity of interferon gamma alone and in combinations with TNFalpha and melphalan in isolated limb perfusion in the BN175 sarcoma tumor model in rats. Oncol Rep 1999;6:173-7. 